\title{
Warped discs and the Unified Scheme
}

\author{
Andrew Lawrence \\ Institute for Astronomy, University of Edinburgh Royal Observatory, Blackford Hill, \\ Edinburgh EH9 3HJ, UK \\ email: al@roe.ac.uk
}

\begin{abstract}
The standard torus picture for explaining the difference between Type I and Type II AGN is physically unlikely and provides no natural explanation for a number of simple facts - the average covering factor, the broad range of covering factors, and the characteristic reprocessing distance. Parsec scale warped discs are a strong alternative. A very simple "misaligned disc" model produces good agreement with covering factor statistics, but predicts too many off-axis emission line cones. I discuss possible ways to improve such a model.
\end{abstract}

\section{Introduction}

The standard Unified Scheme for AGN includes a central continuum source assumed to be an accretion disc around a black hole; a region somewhat further out emitting broad emission lines ; a dusty rotating "torus" beyond this; and gas emitting narrow emission lines on large scales, ionised through the open cone of the torus. (Antonucci and Miller 1985; Krolik and Begelman 1988). Type II AGN are those seen sideways through the obscuring torus, so that one sees only the narrow lines and the IR emission produced by reprocessing of the continuum source by the torus. The torus is assumed to be geometrically thick $(H / R \sim 1)$ and to produce both optical extinction and X-ray absorption. In order to explain the X-ray background, it is popular to assume that most AGN ( 80\%) are obscured. The Unified Scheme has good general observational evidence (polarisation mirrors, and emission line cones, seen in some objects; the relative radio lobe sizes of broad and narrow line radio galaxies; the different relative orientations of polarisation and radio axis in broad and narrow line radio galaxies). However, in detail, the simple scheme has several problems.

\section{Unsatisfactory aspects of the simple torus picture}

(i) The idea of a geometrically thick rotating torus is problematic because vertical motions would need to be of the same order as rotational motions, which on the scales concerned would be around $1000 \mathrm{~km} / \mathrm{s}$. As the expected temperature is $\sim 200 \mathrm{~K}$, the material would be highly supersonic. Discrete gas clouds could be in macroscopic motion, but many papers have argued that such a structure would rapidly dissipate to a flat disc.

(ii) Some statistical differences between Type I and II AGN populations cannot be explained by seeing the same structure at different angles. Circumnuclear imaging shows that on average Type II AGN are dustier and have more disturbed morphologies (Malkan, Gorjian and Tam 1998; Hunt and Malkan 2004). For a given radio power Type II AGN have weaker narrow-line OIII emission (Whittle 1985; Jackson and Browne 1990; Lawrence 1991; Grimes et al. 2004). Assuming radio power indicates true pre-obscured nuclear power, and relative OIII strength is proportional to the uncovered solid angle, there must be a wide range of covering factors; objects with large covering factors are more likely to appear as Type II and possibly also to appear dusty. The mean ratio of 
OIII/radio for Type I vs Type II is $R_{O I I I} \sim 4$ (Grimes et al. 2004 for radio galaxies; by inspection from the data of Whittle 1985 for Seyfert galaxies). The standard torus model has no natural explanation for the distribution of covering factors.

(iii) The simple torus model makes no simple prediction for the mean covering factor, let alone the distribution of covering factors. The correct 'quasar fraction' $f_{Q}$ is in fact still a contentious issue. Radio, X-ray, and OIII selected samples show $f_{Q}$ varying with luminosity from $\sim 0.5$ at high power to $\sim 0.2$ at low power (e.g. Lawrence 1991, Ueda et al. 2003, Simpson 2005). However, IRAS selected mid-IR samples show Type I and II AGN in equal numbers over all luminosities (e.g. Rush, Malkan and Spinoglio 1993), and early Spitzer analyses seem to be giving a similar answer (Lacy et al. 2004). MartinezSansigre (2005) have argued from observations of faint Spitzer sources that most high redshift quasars are obscured, but depending on various assumptions their analysis is consistent with anything in the range $f_{Q} \sim 0.2-0.8$. Willott et al. (2000) noted that considering only high excitation narrow line radio galaxies removes the luminosity dependence, giving $f_{Q} \sim 0.4$, and suggested that low excitation objects, which constitute the majority of the low luminosity narrow line radio galaxies, are a different phenomenon - possibly 'switched off' quasars rather than obscured quasars. (A similar analysis of Xray selected samples would be of great interest.) Another clue to the covering factor is the observation that the luminosity of the IR-bump in quasars is on average $30 \%$ of the luminosity of the UV bump (Sanders et al. 1989). Note however that if there is a range of covering factors, the averaged reprocessed fraction $f_{R}$ for objects seen as Type $\mathrm{I}$ is is not the same as the average covering factor for all AGN.

(iv) The simple torus model makes no simple prediction for the distance at which most extinction/reprocessing takes place. The mean quasar SED (Sanders et al. 1989; Elvis et al. 1994) shows a clear peak at $\sim 10 \mu \mathrm{m}$ corresponding to $T \sim 200 \mathrm{~K}$, much cooler than the dust sublimation temperature, and corresponding to around 1pc in Seyferts, and 10pc in quasars. It has been noted that this is roughly the 'sphere of influence' of the black hole (Krolik and Begelman 1988) but it is not clear why a 'wall of dust' should occur at this distance. Although there is a fairly well defined typical distance/dust temperature, the breadth of the IR bump shows that there is dust at a wide range of temperatures.

In conclusion, we ideally want a model that naturally produces (a) a geometrically thick structure, (b) mean values of $f_{Q} \sim 0.4$ and $f_{R} \sim 0.3$, (c) a distribution of covering factors $N(C)$ such that $R_{O I I I} \sim 4$, and (d) a wide range of dust temperatures, but peaking at $T \sim 200 K$.

\section{A simple re-aligning disc model}

It has been proposed in the past that warped dust discs may be a good way to explain the IR emission in quasars (Phinney 1989; Sanders et al. 1989), as they can cover a significant fraction of the sky, and naturally produce a wide range of temperatures the inner radii do not obscure the outer radii. Can a warped disc model produce the required distribution of covering factors $N(C)$ ? Warped discs clearly occur frequently on kpc scales, but the degree of warping is relatively modest, and it is not clear what can happen on pc scales, although there are plausible mechanisms such as the Pringle instability (Pringle 1996, 1997), tumbling bars (Tohline and Osterbrock 1982) or perhaps torques from large scale magnetic fields.

Rather than trying to produce a physical model, I consider here a very general suggestion: that the angular momenta of the incoming disc and the nuclear disc are completely unconnected. The radio axes of AGN seemed to be aligned at random with respect to their host galaxies (Ulvestad and Wilson 1984; Clarke, Kinney and Pringle 1998; Nagar and 
Wilson 1999; Kinney et al. 2000) so this is a quite reasonable hypothesis. The probability density for the difference $\theta$ of the angular momentum vectors is then $d P=\frac{1}{2} \sin \theta d \theta$. (This assumes $\theta$ can be anywhere from 0 to $\pi$, i.e. the incoming disc can even be counterrotating with respect to the nuclear disc.) At some critical radius some unspecified torque causes the disc to re-align. The covering factor $C$ then depends on the degree of precession during the re-alignment. Complete precession will effectively make an equatorial wall of height $\theta$ giving $C=\sin \theta$. A warp which tilts but doesn't twist at all gives $C=\theta / \pi$ for small $\theta$ (Phinney 1989; the original paper states $C=\theta / 3$ but Phinney has confirmed privately that $\theta / \pi$ is correct). For either of these cases (precessed disc and tilted disc) one can then calculate $N(C)$, for all objects or just for those seen as Type I or II. Likewise, calculating $N(1-C)$ for each Type gives the distribution of relative OIII strengths.

For precessed discs, any value $\theta>\pi / 2$ (i.e. counter-rotating incoming discs) gives $C=1$, predicting that half of all AGN are completely obscured and possibly haven't even been recognised. Even for objects with $\theta<\pi / 2$, the distribution is peaked towards $C=1$. The quasar fraction comes from integrating under $N_{I}(C)$ for Type I only and gives $f_{Q}=0.22$ or $f_{Q}=0.11$ including the completely obscured objects, significantly in disagreement with observations. The average reprocessed fraction for quasars is $f_{R} \sim 0.5$, which is somewhat too large. Taking the median values of $N(1-C)$ for Types I and II we get $R_{\text {OIII }} \sim 5$, but the distribution of relative OIII values, piled up towards low OIII for Type IIs, does not resemble the observations. This very simplest model therefore does not work.

For tilted discs $N(C)$ peaks around $C=0.5$. The quasar fraction found from integrating under $N_{I}(C)$ for Type I only gives $f_{Q}=0.48$. The typical reprocessed fraction for quasars comes from the mean of $N_{I}(C)$ which is $f_{R} \sim 0.35$. The ratio of typical relative OIII strengths comes from comparing the peaks of $N_{I}(1-C)$ and $N_{I I}(1-C)$ from which we find $R_{O I I I} \sim 2$. So a tilted re-aligned disc, on these statistical grounds, works fairly well - it gives good agreement with observations for $f_{Q}$ and $f_{R}$ and somewhat too small a difference between Type I and II for $R_{O I I I}$. The covering factor equation $C=\theta / \pi$ is strictly only correct for small $\theta$, so this agreement may be somewhat fortuitous. However there are other reasons why a tilted disc with no twist cannot be the correct model. On a given side of the inner disc, the warped outer disc is not azimuthally symmetric, and so does not produce simple shadow-cones. Seen in projection, it will produce bipolar cone-like ionisation structures, but they will be strongly one-sided, and will not usually be aligned with the radio axis, whereas observations do seem to show such an alignment (Schmitt et al. 2003).

So, for different reasons, simple versions of both twisted and untwisted warped disc models don't quite work. The conclusion could be that warped discs are not the answer; or it could be that the observed cones are not 'shadow cones' but rather are 'matter cones' formed by an outflow. Finally of course, one could relax the strict assumptions of the simplest model. The degree of twist could be between the extremes of no twist at all and complete precession. The radiation instability model of Pringle (1997) produces such a partial twist, but still has a problem with jets and cones not aligning. Alternatively, it could be that the axis difference $\theta$ between the outer and inner disc is not random. Analysis by Schmitt et al. (2002) finds that for radio galaxies the difference between the radio axis and the axis of the kpc scale dust disc is not random over all solid angle, but over a large polar cap. This is probably the most obvious variant to explore next. 


\section{Conclusions}

The standard torus model has some significant problems, and lacks any natural prediction for the average covering factor or the distribution of covering factors. A simple re-aligning disc model, where the incoming disc and the nuclear disc are unrelated, makes quite simple and definite predictions, depending on whether the incoming disc twists as it re-aligns.

Based on the simple numerical tests of the previous section, the precessed disc does not fit the facts, whereas the tilted disc fits quite well. However, the tilted disc model predicts ionisation structures that are not in general aligned with the nuclear axis, and so can almost certainly be ruled out.

Perhaps the most promising possibility is that there is a fully precessed re-aligned disc, but that the difference between incoming and nuclear discs is not completely random. This possibility will be explored in a fuller version of this work.

\section{Acknowledgements}

Thanks to Martin Elvis and Andrew King, with whom I have many related discussions over several years.

\section{References}

Antonucci R. R. J. \& Miller A. S. 1985, ApJ, 297, 621

Clarke C. J., Kinney A. L. \& Pringle J. E. 1998, ApJ, 495, 189

Elvis M., Wilkes B. J., McDowell J. C., Green R. F., Bechtold J., Willner S. P. et al. 1994, ApJSS, 95, 1

Grimes J. A., Rawlings S. \& Willott C. J. 2004, MNRAS, 349, 503

Hunt L. K. \& Malkan M. A. 2004, ApJ, 616, 707

Jackson N. \& Browne I. W. A. 1990, Nature, 343, 43

Kinney A. L., Schmitt H. R., Clarke C. J., Pringle J. E., Ulvestad J. S. \& Antonucci R. R. J. 2000, ApJ, 537, 152

Krolik J. H. \& Begelman M. C. 1988, ApJ, 329, 702

Lawrence A. 1991, MNRAS, 252, 586

Lacy M., Storrie-Lombardi L. J., Sajina A. et al. 2004, ApJ, 154, 166

Malkan M. A. Gorjian V. \& Tam R. 1998, ApJSS, 117, 25

Martinez-Sansigre A., Rawlings S., Lacy M., Fadda D., Marline F. R., Simpson C. et al. 2005, Nature, 436, 666

Nagar N. M. \& Wilson A. 1999, ApJ, 516, 97

Phinney E. S. 1989, in Theory of Accretion Disks, p. 457, eds F.Meyer et al. (Dordrecht: Kluwer)

Pringle J. E. 1996, MNRAS, 281, 357

Pringle J. E. 1997, MNRAS, 292, 136

Rush B., Malkan M. A. \& Spinoglio L. 1993, ApJSupp 89, 1

Sanders D. B., Phinney E. S., Neugebauer G., Soifer B. T. \& Mathews K. 1989, ApJ, 347, 29

Schmitt H. R., Pringle J. E., Clarke C. J. \& Kinney A. L. 2002, ApJ, 575, 150

Schmitt H. R., Donley J. L., Antonucci R. R. J., Hutchings J. B. \& Kinney A. L. 2003, ApJSS, 148,327

Simpson C. 2005, MNRAS, 360, 565

Tohline J. E. \& Osterbrock D. E. 1982, ApJ, 252, L49

Ulvestad J. S. \& Wilson A. S. 1984, ApJ, 285, 439

Ueda Y., Akiyama M., Ohta K. \& Miyaji T. 2003, ApJ, 598, 886

Whittle M. 1985, MNRAS, 213, 33

Willott C. J., Rawlings S., Blundell K. \& Lacy M. 2000, MNRAS, 316, 449 
FuKun LiU: Comment: The warped disc model has problems caused by radiation instability of Pringle, as you mentioned. My comment is that the disc warps are not due to radiation, but due to interaction of an inclined supermassive black hole binary with a standard accretion disc, which is the natural consequence of hierarchical galaxy formation model and has been investigated and introduced to explain the observations of radio jet morphology (Liu 2004, MNRAS). If this is the case, the difficulties with warped disc model may be overcome.

Giorgio MATT: Can the warped disc model explain the mismatch between optical and X-ray classification occurring in $\sim 10 \%$ of AGN in the local universe?

ANDREW LAWREnCE: The warped disc model per se is irrelevant to this problem - I was simply pointing out that many papers draw naive conclusions because they are not careful about distinguishing between X-ray absorption and optical extinction, which are often unconnected. 


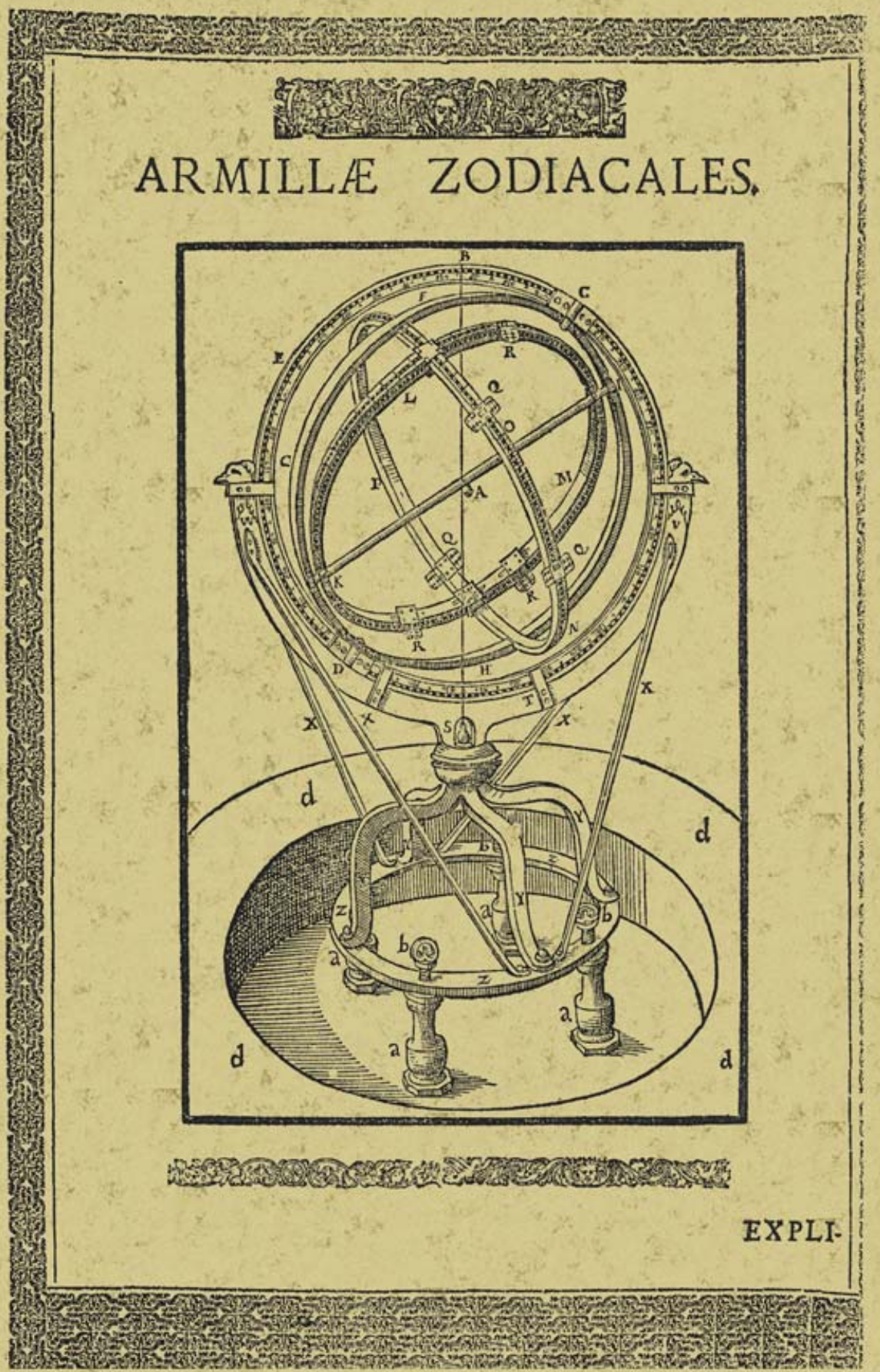

